\title{
The Protective Effect of Compound Danshen Dripping Pills on Oxidative Stress after Retinal Ischemia/Reperfusion Injury in Rats
}

\author{
Bingwen Lu, Xingwei $\mathrm{Wu}^{*}$ \\ Ophthalmology Department, Shanghai First People's Hospital, Shanghai, China \\ Email: ${ }^{* 704487389 @ q q . c o m}$
}

Received 19 March 2015; accepted 9 May 2015; published 12 May 2015

Copyright (C) 2015 by authors and Scientific Research Publishing Inc.

This work is licensed under the Creative Commons Attribution International License (CC BY).

http://creativecommons.org/licenses/by/4.0/

(c) (7) Open Access

\begin{abstract}
Objective: To investigate the effect of Compound Danshen Dripping Pills (CDDP) on oxidative stress after ischemia/reperfusion (I/R) injury in the rat retina. Methods: Adult male SD rats were randomly divided into 3 groups: sham (group A), I/R (group B), and I/R plus CDDP (group C). Retinal ischemia/reperfusion injury (RIRI) was introduced by increasing the intraocular pressure (IOP) to $110 \mathrm{mmHg}$ for $60 \mathrm{~min}$ via cannulation into the anterior chamber. Right after the insult, CDDP was administered intragastrically $(450 \mathrm{mg} / \mathrm{kg} / \mathrm{d})$ for 7 days. The levels of malondialdehyde (MDA), the activities of superoxide dismutase (SOD), glutathione peroxidase (GSH-Px), and catalase (CAT) in the retinal tissues were determined on $\mathrm{d} 1$ and $\mathrm{d} 7$ after the ischemic insult. Results: Following ischemia, the MDA levels in group $B$ and group $C$ were significantly higher than those in group $A$ $(p<0.01)$. CDDP significantly lowered MDA levels in group $C$ when compared with group $B(p<$ 0.01). The activities of SOD, GSH-Px and CAT were higher in group $A$ than in group $B$ and group $C$ $(p<0.01)$. CDDP could increase the activities of SOD, GSH-Px and CAT remarkably in group $C$ when compared with group B $(p<0.01)$. Conclusion: CDDP can protect the retina from I/R injury through reducing oxidative stress, and thus may be a promising method for the treatment of ischemic retinal disorders.
\end{abstract}

\section{Keywords}

Compound Danshen Dripping Pills (CDDP), Retinal Ischemia/Reperfusion Injury (RIRI), Oxidative Stress, Rats

\section{Introduction}

Ischemia/reperfusion (I/R) occurs in many clinical conditions and can seriously impair the affected organ or tis-

"Corresponding author.

How to cite this paper: Lu, B.W. and Wu, X.W. (2015) The Protective Effect of Compound Danshen Dripping Pills on Oxidative Stress after Retinal Ischemia/Reperfusion Injury in Rats. Chinese Medicine, 6, 90-96.

http://dx.doi.org/10.4236/cm.2015.62010 
sues [1]. Retinal ischemia/reperfusion injury (RIRI) is a common cause of visual impairment and blindness. Central retinal artery occlusion (CRAO), central retinal vein occlusion (CRVO), branch retinal artery occlusion (BRAO), branch retinal vein occlusion (BRVO), acute glaucoma, diabetic retinopathy, and age-related macular degeneration (AMD) are all associated with retinal ischemia [2]. The retina is highly dependent on its oxygen supply, while restoration of blood flow after ischemia paradoxically initiates more serious tissue injury and cell death, leading to an irreversible impairment of retinal function [2]. Therefore, the management of RIRI is crucial.

Compound Danshen Dripping Pills (CDDP) is a Chinese patent medicine, developed on the basis of Traditional Chinese Medicine (TCM) theory and modern preparation technologies. It is widely used in the prevention and treatment of coronary arteriosclerosis, angina pectoris, hyperlipidemia, and other cardiovascular diseases in China [3]. This new drug compound mainly consists of salvia miltiorrhiza and panax notoginseng [4]. In the past few decades, CDDP has been demonstrated to have antioxidant, anti-inflammatory properties, and it can also protect endothelial function, inhibit platelet adhesion as well as improve microcirculation [5]-[8]. Because of its characters of multi-components, multi-effects, and multi-targets, CDDP went through phase II clinical research in USA in 2010 [9]. Although many studies have provided evidences that CDDP is I/R protective in cardiovascular disorders, its potential protective effects in ameliorating retina ischemic disorders have received little attention. Thus, we hypothesized that CDDP could have some therapeutic efficacy on RIRI. In our previous study, we have already demonstrated that CDDP can exert protective effects on the retina under ischemic conditions through examining resultant ERG and histological findings [10].

I/R-induced oxidative stress is thought to be the direct cause of retinal injury. MDA, a degraded product of lipid peroxidation, produces cytotoxicity by reacting with the amino of nucleic acid [11], whereas SOD, GSH-Px and CAT are major scavengers of reactive oxygen species (ROS), which are known to trigger apoptosis and are massively generated during I/R [12]. Previous study showed that changes of MDA levels and SOD activities were indicators for lipid peroxidation degrees and therefore reflected the severity of tissue damage [13]. Therefore, in the present study, in order to elucidate whether CDDP exerts protective effects on the retinal under ischemic conditions against oxidative stress, we examined the MDA level and the activities of SOD, GSH-Px, and CAT in the retina tissues.

\section{Materials and Methods}

\subsection{Research Objects and Grouping}

Fifty-four adult male SD rats, weighing from 200 to $350 \mathrm{~g}$, were provided by Joinn Laboratories (Suzhou). The animal care strictly conformed to the Association for Research in Vision and Ophthalmology Statement for the Use of Animals in Ophthalmology and Vision Research. The animals were housed under controlled conditions: a 12-hour light/dark cycle; a temperature of $20^{\circ} \mathrm{C}$ to $26^{\circ} \mathrm{C}$, and humidity in the range of $40 \%$ to $70 \%$. The animals had free access to standard food and drinking water.

Rats were randomly divided into three groups: sham (group A) $(\mathrm{n}=18), \mathrm{I} / \mathrm{R}$ (group B) $(\mathrm{n}=18)$, and I/R plus CDDP (group C) $(\mathrm{n}=18)$.

\subsection{Experiment Medicine}

CDDP was provided by Tasly Pharmaceutical Co., Ltd. (Tianjin, China). The CDDP was dissolved in a certain amount of water to a certain concentration of per milliliter of drug solution. Group C was treated with drug solution at a dose of $450 \mathrm{mg} / \mathrm{kg} / \mathrm{d}$ for seven days. Meanwhile, Group A and Group B were treated with the same volume of $0.9 \%$ saline solution.

\subsection{Experiment Methods}

\subsubsection{Establishment of RIRI Model in Rats}

Animals were anesthetized intraperitoneally with $1 \%$ pentobarbital sodium $(0.7 \mathrm{ml} / 100 \mathrm{~g})$. Body temperature was maintained at $36.5^{\circ} \mathrm{C}-37^{\circ} \mathrm{C}$ using a heated blanket. For corneal analgesia, one drop of $0.4 \%$ oxybuprocaine hydrochloride was used. Pupillary dilation was achieved with $0.5 \%$ tropicamide and $0.5 \%$ phenylephrine.

Retinal ischemia was induced by increasing the IOP in the right eye to $110 \mathrm{mmHg}$ for $60 \mathrm{~min}$. After dilation of the pupil, the anterior chamber of the right eye (only the right eye was used experimentally) was cannulated with a 30-gauge needle connected to a physiological saline bag. The IOP was raised to $110 \mathrm{mmHg}$ by keeping 
the bag at $150 \mathrm{~cm}$ above the eye. Retinal ischemia was confirmed when there was whitening of the iris and loss of the red reflex of the retina. After $60 \mathrm{~min}$ of ischemia, the needle was removed and the IOP was returned to normal pressure. For the sham group, the procedure was performed without elevating the saline bag.

\subsubsection{Determination of the Levels of MDA and Activities of SOD, GSH-Px, and CAT}

The levels of MDA and the activities of SOD, GSH-Px, and CAT were determined in each retina 1 day and 7 days after the ischemic insult.

The retina samples were prepared as a $10 \%$ homogenate in $0.9 \%$ saline using a homogenizer on ice according to their respective weight. Then, the homogenate was centrifuged at $3500 \mathrm{rpm}$ for $15 \mathrm{~min}$, and the supernatant was collected and diluted.

MDA levels were determined using the double heating method. Briefly, $2.5 \mathrm{ml}$ of thiobarbituric acid (TBA) means thiobarbituric acid solution $(100 \mathrm{~g} / \mathrm{L})$ was added to $0.5 \mathrm{~mL}$ of homogenate in each centrifuge tube, and the tubes were placed in boiling water for $15 \mathrm{~min}$. After cooling with flowing water, the tubes were centrifuged at $1000 \mathrm{rpm}$ for $10 \mathrm{~min}, 2 \mathrm{~mL}$ of the supernatant was added to $1 \mathrm{~mL}$ of the TBA solution $(6.7 \mathrm{~g} / \mathrm{L})$, and the tube was placed in boiling water for another $15 \mathrm{~min}$. After cooling, the amount of thiobarbituric acid-reactive species was measured at $532 \mathrm{~nm}$. The MDA concentration was calculated from the absorbance coefficient of the MDATBA complex.

SOD activity was determined through the inhibition of nitrotetrazolium blue (NTB) reduction by the xanthine/ xanthine oxidase system as a superoxide generator. The activity was assessed in the supernatant after the addition of $1.0 \mathrm{~mL}$ of ethanol/chloroform $(5 / 3, \mathrm{v} / \mathrm{v})$ to the same volume of sample and centrifugation for $15 \mathrm{~min}$ at $3000 \mathrm{rpm}$. The production of formazan was determined at $560 \mathrm{~nm}$. One unit of SOD activity was defined as the amount of protein that inhibited the rate of NTB reduction by $50 \%$.

GSH-Px activity was measured by the method of Paglia and Valentine. The enzymatic reaction in the tube that contained reduced nicotinamide adenine dinucleotide phosphate, reduced glutathione, sodium azide, and glutathione reductase was initiated by the addition of $\mathrm{H}_{2} \mathrm{O}_{2}$, and the change in absorbance at $340 \mathrm{~nm}$ was monitored by spectrophotometry. The data are expressed as $\mathrm{U} / \mathrm{g}$ of protein.

CAT activity was measured by the method of Cohen. The principle of the assay was based on the determination of the rate constant ( $\mathrm{s}-1, \mathrm{k}$ ) of $\mathrm{H}_{2} \mathrm{O}_{2}$ decomposition. The rate constant of the enzyme was determined by measuring the absorbance change per minute. The data are expressed as $\mathrm{k} / \mathrm{g}$ of protein.

\subsection{Statistical Methods}

Data were represented as the mean \pm SEM. To determine the significance of differences, analysis of variance (ANOVA) were carried out using SPSS 18.0 software. P values less than 0.05 were considered statistically significant in this study.

\section{Results}

\subsection{Effects of CDDP on the MDA Level and Activities of SOD, GSH-Px, and CAT on d1 after RIRI}

To estimate the anti-oxidative effect of CDDP, the MDA level and the activities of SOD, GSH-Px, and CAT of the retina tissue were measured on $\mathrm{d} 1$ after I/R injury. As a marker of lipid peroxidation, the MDA level in the retina tissues of group B was remarkably higher than that in group A $(p<0.01)$. CDDP significantly inhibited MDA production in the retina tissue after RIRI when compared with group $B(p<0.01)$. Data are shown in Figure 1(a).

SOD, GSH-Px, and CAT are pivotal enzymes scavenging ROS in vivo. The activities of SOD, GSH-Px, and CAT were greatly reduced in group B than in goup A after I/R injury $(p<0.01)$. Compared with group B, CDDP could significantly increase the activities SOD, GSH-Px, and CAT $(p<0.01)$. Data are shown in Figures 1(b)-(d), respectively.

\subsection{Effects of CDDP on the MDA Level and Activities of SOD, GSH-Px, and CAT on d7 after RIRI}

Seven days after RIRI, the MDA level in the group B was lower than those of day 1 . However, compared with 


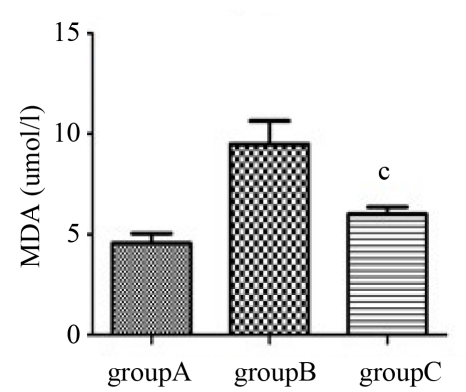

(a)

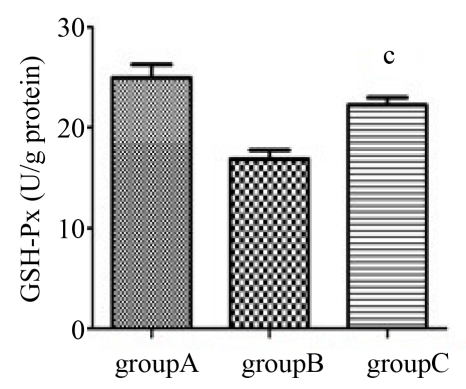

(c)

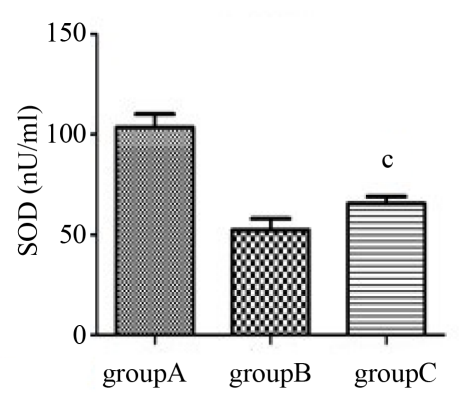

(b)

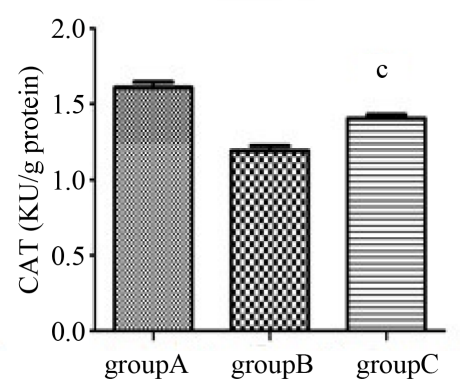

(d)

Figure 1. Effects of CDDP on MDA levels and the activities of SOD, GSH-Px and CAT on the retina tissue from I/R-induced retinal injury on d1. Data are expressed as mean \pm SD and are representative of 3 experiments $(n=9)$. ${ }^{c} p<0.01$ vs. group $B$ with group $C$.

group $\mathrm{C}$, the MDA levels were still significantly higher in group B $(\mathrm{p}<0.01)$. Data are shown in Figure 2(a). The activities of SOD, GSH-Px, and CAT were higher than those of d1. Compared with group C, the activities were still significantly lower in the group B $(\mathrm{p}<0.01)$. Data are shown in Figures $2(\mathrm{~b})-(\mathrm{d})$, respectively.

\section{Discussion}

The present study was performed to evaluate the protective effects of CDDP against oxidative stress on the retina following RIRI in the rats. We found that CDDP improved the oxidative parameters of the retinal tissue in the I/R model, which suggested that CDDP might be a potential choice for the treatment of RIRI-related eye diseases.

A number of ocular diseases have been associated with retinal ischemia reperfusion injury, including retinal vascular occlusion, acute glaucoma, diabetic retinopathy, and retinopathy of prematurity [14]. Oxygen-free radicals [15], endogenous excitatory amino acids [16], calcium imbalances [17], and nitric oxide [18] [19], are involved in the pathogenesis of $\mathrm{I} / \mathrm{R}$ injury. The exact mechanism of RIRI is complicated, mediated by a variety of factors, which makes it a hot pot problem around the world. Accordingly, finding a way to reduce or prevent RIRI, thus reduce visual impairment is of great importance.

Oxygen is crucial for life and metabolic processes, however, reactive metabolites of oxygen can be toxic to cells [20] [21]. Particularly, the sudden reintroduction of oxygen into tissues during reperfusion may exacerbate the cellular damage secondary to ischemia. ROS is considered to be a critical mediator of ischemia reperfusion injury. The generation of excess ROS can affect subcellular structures and functions, resulting in mitochondrial dysfunction, lipid peroxidation of polyunsaturated fatty acids in membranes, and alterations in protein or DNA structure [21]-[23].

Abundant studies of retinal tissue have shown that oxidative stress was an fundamental factor in cellular damage during IR injury. MDA is a naturally occurring product of lipid peroxidation, a process in which unsaturated fatty acids are oxidized to form radicals [24]. The level of MDA is currently used to assess oxidative tissue damage after I/R injury [24]. Free radicals produced in living organisms can be scavenged by intrinsic antioxidant enzymes, including SOD, CAT, and GSH-Px, thus, the activities of these enzymes may reflect the anti- 


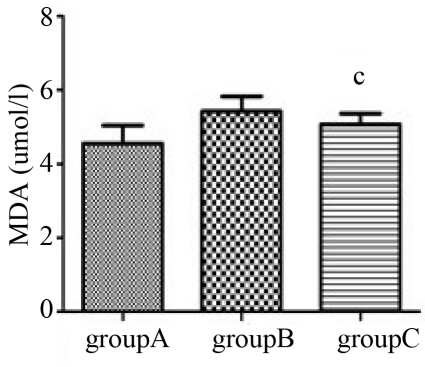

(a)

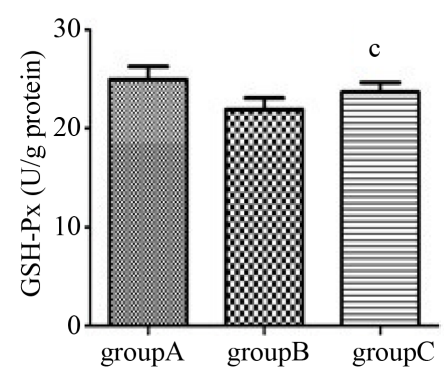

(c)

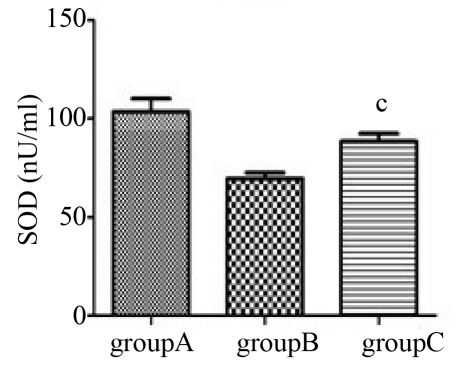

(b)

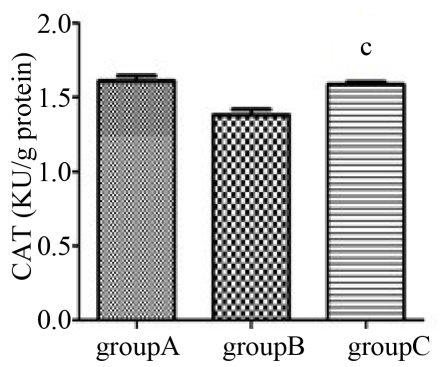

(d)

Figure 2. Effects of CDDP on MDA levels and the activities of SOD, GSH-Px and CAT on the retina tissue from I/R-induced retinal injury on d7. Data are expressed as mean \pm SD and are representative of 3 experiments $(n=9)$. ${ }^{c} p<0.01$ vs. group B with group $C$.

oxidative ability of the body [25]-[27].

Therefore, the aim of this study is to investigate the protective effects of CDDP against oxidative stress on the retina following RIRI in the rats through examining the MDA level and the activities of SOD, GSH-Px, and CAT in the retina tissues.

In the present study, we examined the changes in the levels of MDA, SOD, CAT, and GSH-Px, to elucidate the mechanism by which CDDP protects retinal cell injuries from oxidative stress. The results of $\mathrm{d} 1$ and $\mathrm{d} 7$ after RIRI have shown that $\mathrm{I} / \mathrm{R}$ injury resulted in a significant increase in the MDA levels and decrease in the activities of SOD, CAT, and GSH-Px in the retina. However, CDDP could significantly decrease the MDA level and enhance the antioxidant enzyme activity in retinal tissue, implying that CDDP ameliorated the injury. The anti-oxidative stress effect of CDDP may be part of its protective mechanisms in RIRI.

CDDP is a new drug compound preparation in treating cardiovascular diseases. The chemical analysis of CDDP reported previously shows that the main ingredients are salvia miltiorrhiza and panax notoginseng [4]. One pill of CDDP (obtained from Tasly Pharmaceutical, Tianjin, China) contains $9 \mathrm{mg}$ of salvia miltiorrhiza and $1.76 \mathrm{mg}$ of panax notoginseng. Salvia miltiorrhiza could improve cardiac function through improving antioxidant activity [5], and through preventing cardiomyocyte apoptosis [6]. Panax notoginseng could protect against myocardial damage and decrease lipid peroxidation [7]. With the combination of multiple components, CDDP hits multiple targets, and exerts the synergistic therapeutic effects. CDDP has been reported to be effective in the prevention of tissue damage in the heart and brain after IR injury, whereas there are few studies on the protective effect of CDDP against retinal damage caused by $\mathrm{I} / \mathrm{R}$, which is commonly seen in clinical practice. Thus, our studies investigated the protective effect and the underlying mechanisms.

In this study, the dose of CDDP chosen was the high-dose $(450 \mathrm{mg} / \mathrm{kg} / \mathrm{d})$ used in the Li et al. study [28]. Our previous study demonstrated that the high-dose CDDP $(450 \mathrm{mg} / \mathrm{kg} / \mathrm{d})$ could exert protective effects on the retina under ischemic conditions through examining resultant ERG and histological findings [10]. Those positive results provided proof for the use of the dosage of CDDP.

However, it should be noted that the anti-oxidative stress effect may not be the sole protective mechanism of CDDP. Various other mechanisms, such as anti-inflammatory and anti-apoptosis activities, could also play a role in the observed protective effects of CDDP during I/R injury. Further investigations should be carried out to 
clarify this.

\section{Conclusion}

In conclusion, the present study demonstrates that CDDP is effective at protecting the retina during I/R injury. CDDP protected the retina from IR injury, possibly via a mechanism involving the regulation of oxidative parameters.

\section{Acknowledgements}

This work was supported by a grant from Shanghai First People's Hospital. The author was grateful to Professor Wu from Shanghai First People's Hospital for final edict of this manuscript.

\section{References}

[1] Grace, P.A. (1994) Ischemia-Reperfusion Injury. British Journal of Surgery, 81, 637-647. http://dx.doi.org/10.1002/bjs.1800810504

[2] Osborne, N.N., Casson, R.J., Wood, J.P., Chidlow, G., Graham, M. and Melena, J. (2004) Retinal Ishchemia: Mechanisms of Damage and Potential Therapeutic Strategies. Progress in Retinal and Eye Research, 23, 91-147. http://dx.doi.org/10.1016/j.preteyeres.2003.12.001

[3] Jia, Y., Huang, F., Zhang, S. and Leung, S.W. (2012) Is Danshen (Salvia miltiorrhiza) Dripping Pill More Effective than Isosorbide Dinitrate in Treating Angina Pectoris? A Systematic Review of Randomized Controlled Trials. International Journal of Cardiology, 157, 330-340. http://dx.doi.org/10.1016/j.ijcard.2010.12.073

[4] Zheng, X.H., Zhao, X.F., Zhao, X., Wang, S.X., Wei, Y.M. and Zheng, J.B. (2007) Determination of the Main Bioactive Metabolites of Radix Salvia miltiorrhizae in Compound Danshen Dripping Pills and the Tissue Distribution of Danshensu in Rabbit by SPE-HPLC-MS ${ }^{\mathrm{n}}$. Journal of Separation Science, 30, 851-857. http://dx.doi.org/10.1002/jssc.200600287

[5] Wang, S.B., Tian, S., Yang, F., Yang, H.G., Yang, X.Y. and Du, G.H. (2009) Cardioprotective Effect of Salvianolic Acid A on Isoproterenol-Induced Myocardial Infarction in Rats. European Journal of Pharmacology, 615, 125-132. http://dx.doi.org/10.1016/j.ejphar.2009.04.061

[6] Li, M., Zhao, M.Q., Kumar Durairajan, S.S., Xie, L.X., Zhang, H.X., Kum, W.F., Goto, S. and Liao, F.L. (2008) Protective Effect of Tetramethylpyrazine and Salvianolic Acid B on Apoptosis of Rat Cerebral Microvascular Endothelial Cell Under High Shear Stress. Clinical Hemorheology and Microcirculation, 38, 177-187.

[7] Ruan, J.Q., Leong, W.I., Yan, R. and Wang, Y.T. (2010) Characterization of Metabolism and in Vitro Permeability Study of Notoginsenoside R1 from Radix Notoginseng. Journal of Agricultural and Food Chemistry, 58, 5770-5776. http://dx.doi.org/10.1021/jf1005885

[8] Yuan, R.Y. and Li, G.P. (2009) Multi-Target Effects of Compound Danshen Dripping Pills in Prevention and Treatment of Cardiovascular Diseases. Chinese Journal of New Drugs, 18, 377-380.

[9] Anonymous (2010) Compound Danshen Dripping Pills Passed Phase II Clinical Trials by FDA. China Journal of Pharmaceutical Economics, 4, 94.

[10] Lu, B.-W. and Wu, X.-W. (2014) Protective Effects of Compound Danshen Dripping Pills on Retinal Ischemia-Reperfusion Injury in Rats. Recent Advances in Ophthalmology, 34, 1030-1034.

[11] Banin, E., Berenshtein, E., Kitrossky, N., Pe'er, J. and Chevion, M. (2000) Gallium-Desferrioxamine Protects the Cat Retina against Injury after Ischemia and Reperfusion. Free Radical Biology and Medicine, 28, 315-323. http://dx.doi.org/10.1016/S0891-5849(99)00227-0

[12] Anderson, R.E., Maude, M.B. and Neilsen, J.C. (1985) Effect of Lipid Peroxidation on Rhodopsin Regeneration. Experimental Eye Research, 4, 65-71. http://dx.doi.org/10.3109/02713688508999969

[13] Xie, Z., Wu, X., Gong, Y., Song, Y., Qiu, Q. and Li, C. (2007) Intraperitoneal Injection of Ginkgo Biloba Extract Enhances Antioxidation Ability of Retina and Protects Photoreceptors after Light-Induced Retinal Damage in Rats. Current Eye Research, 32, 471-479. http://dx.doi.org/10.1080/02713680701257621

[14] Shibuki, H., Katai, N., Yodoi, J., Uchida, K. and Yoshimura, N. (2000) Lipid Peroxidation and Peroxynitrite in Retinal Ischemia-Reperfusion Injury. Investigative Ophthalmology Visual Science, 41, 3607-3614.

[15] Kwon, Y.H., Rickman, D.W., Baruah, S., Zimmerman, M.B., Kim, C.S., Boldt, H.C., Russell, S.R. and Hayreh, S.S. (2005) Vitreous and Retinal Amino Acid Concentrations in Experimental Central Retinal Artery Occlusion in the Primate. Eye (London), 19, 455-463. http://dx.doi.org/10.1038/sj.eye.6701546

[16] Pannicke, T., Uckermann, O., Iandiev, I., Biedermann, B., Wiedemann, P., Perlman, I., Reichenbach, A. and Bring- 
mann, A. (2005) Altered Membrane Physiology in Müller Glial Cells after Transient Ischemia of the Rat Retina. Glia, 50, 1-11. http://dx.doi.org/10.1002/glia.20151

[17] Toriu, N., Akaike, A., Yasuyoshi, H., Zhang, S., Kashii, S., Honda, Y., Shimazawa, M. and Hara, H. (2000) Lomerizine, a $\mathrm{Ca}^{2+}$ Channel Blocker, Reduces Glutamate-Induced Neurotoxicity and ischemia/Reperfusion Damage in Rat Retina. Experimental Eye Research, 70, 475-484. http://dx.doi.org/10.1006/exer.1999.0809

[18] Yoneda, S., Tanihara, H., Kido, N., Honda, Y., Goto, W., Hara, H. and Miyawaki, N. (2001) Interleukin-1Beta Mediates Ischemic Injury in the Rat Retina. Experimental Eye Research, 73, 661-667. http://dx.doi.org/10.1006/exer.2001.1072

[19] Sanchez, R.N., Chan, C.K., Garg, S., Kwong, J.M., Wong, M.J., Sadun, A.A. and Lam, T.T. (2003) Interleukin-6 in Retinal Ischemia Reperfusion Injury in Rats. Investigative Ophthalmology Visual Science, 44, 4006-4011. http://dx.doi.org/10.1167/iovs.03-0040

[20] Di Mascio, P., Murphy, M.E. and Sies, H. (1991) Antioxidant Defense Systems: The Role of Carotenoids, Tocopherols, and Thiols. The American Journal of Clinical Nutrition, 53, 194S-200S.

[21] Marnett, L.J. (2000) Oxyradicals and DNA Damage. Carcinogenesis, 21, 361-370. http://dx.doi.org/10.1093/carcin/21.3.361

[22] Szabo, M.E., Droy-Lefaix, M.T., Doly, M., Carré, C. and Braquet, P. (1991) Ischemia and Reperfusion-Induced Histologic Changes in the Rat Retina. Eemonstration of a Free Radical-Mediated Mechanism. Investigative Ophthalmology Visual Science, 32, 1471-1478.

[23] Nayak, M.S., Kita, M. and Marmor, M.F. (1993) Protection of Rabbit Retina from Ischemic Injury by Superoxide Dismutase and Catalase. Investigative Ophthalmology Visual Science, 34, 2018-2022.

[24] Del Rio, D., Stewart, A.J. and Pellegrini, N. (2005) A Review of Recent Studies on Malondialdehyde as Toxic Molecule and Biological Marker of Oxidative Stress. Nutrition, Metabolism Cardiovascular Diseases, 15, 316-328. http://dx.doi.org/10.1016/j.numecd.2005.05.003

[25] Chance, B., Sies, H. and Boveris, A. (1979) Hydroperoxide Metabolism in Mammalian Organs. Physiological Reviews, 59, 527-605.

[26] Crack, P.J., Taylor, J.M., de Haan, J.B., Kola, I., Hertzog, P. and Iannello, R.C. (2003) Glutathione Peroxidase-1 Contributes to the Neuroprotection Seen in the Superoxide Dismutase-1 Transgenic Mouse in Response to Ischemia/Reperfusion Injury. Journal of Cerebral Blood Flow Metabolism, 23, 19-22. http://dx.doi.org/10.1097/00004647-200301000-00002

[27] Liochev, S.I. and Fridovich, I. (2002) Superoxide and Nitric Oxide: Consequences of Varying Rates of Production and Consumption: A Theoretical Treatment. Free Radical Biology Medicine, 33, 137-141. http://dx.doi.org/10.1016/S0891-5849(02)00864-X

[28] Li, Y.M., Liu, X.Q., Chen, H.Q., Chen, X.A. and Liu, L.Y. (2010) Effect of Fufang danshen diwan on Atherosclerosis and the Level of TAFI in Rats. Chinese Journal of Gerontology, 29, 280-281. 